\title{
Dynamics of Photoinduced Interfacial Electron Transfer and Charge Transport in Dye-Sensitized Mesoscopic Semiconductors
}

\author{
Verner K. Thorsmølle, Bernard Wenger ${ }^{a}$, Joël Teuscher, Christophe Bauer, and Jacques-E. Moser*
}

\begin{abstract}
Molecular systems designed for the conversion of solar energy offer ideal models in the study of the kinetics of light-induced electron transfer at surfaces. Due to their high porosity, nanocrystalline oxide semiconductor films allow investigations of interfacial and lateral charge transfer processes that are barely detectable on flat surfaces. Although it has proven to be very promising, the redox photochemistry of the metal oxide I molecular monolayer I electrolyte interface is still a largely unexplored scientific domain, offering huge potential for investigation and exploitation of physical and chemical processes. Carrier trapping and charge transport are also key to the efficiency of molecular photonic devices. Carrier dynamics and transport in unconventional media are studied utilizing $\mathrm{THz}$ time domain spectroscopy. We summarize here some aspects of the work currently carried out in these fields as part of our continued effort in the fundamental study of the dynamics of photoinduced electron transfer processes.
\end{abstract}

Keywords: Interfacial redox photochemistry · Kinetics of electron transfer · Mesoscopic materials Terahertz time-domain spectroscopy · Ultrafast laser spectroscopy

\section{Introduction}

The use of molecular species to transduce or store signals in opto-electronic devices is becoming one of the most exciting fields of modern science. Numerous applications for such systems can be foreseen, ranging from information storage and imaging to molecular photovoltaics. ${ }^{[1]}$ One particularly intriguing configuration employs a mesoscopic film of a semiconductor oxide as a support for the molecular transducer.

\footnotetext{
${ }^{*}$ Correspondence: Prof. Dr. J.-E. Moser Photochemical Dynamics Group Institute of Chemical Sciences \& Engineering Ecole Polytechnique Fédérale de Lausanne EPFL ISIC GR-MO, Station 6

$\mathrm{CH}-1015$ Lausanne

Tel.: + 41216933628

Fax: +41216934111

E-Mail: je.moser@epfl.ch

aPresent address: CSEM, Centre Suisse

d'Electronique et de Microtechnique SA

CH-2002 Neuchâtel
}

These films constitute a network of nanocrystalline particles such as titania, niobia or zinc oxide, sintered together to allow charge carrier transport to take place. The pores between the nanoparticles are filled with an electrolyte or a solid-state organic hole conductor, forming an interpenetrating heterojunction of very large contact area. As electrons can rapidly percolate through the film, the entire surface-adsorbed molecular layer can be electronically addressed. Charge transfer events involving adsorbed molecules can thus be induced through the nanocrystalline support and recorded as electrical current. Optical monitoring is also facile as the signals arising from the grafted molecules are greatly enhanced due to the huge internal surface area of the junction. Thus, the nanocrystalline oxide films serve as an interface between the molecular- and macroscopic world providing new opportunities to examine light-induced interfacial reactions on a molecular scale.

Mesoporous systems recently gained importance as the basis of new electrochemical devices, such as batteries, sensors, and solar cells. In these applications, results depend strongly upon the properties of charge transport. Carrier mobility and dynamics in nanostructured metal oxide assemblies, amorphous organic hole-transporting media, conjugated polymers and hybrid systems are ideally studied utilizing terahertz time-domain (THz-TDS) and optical pump-THz probe (OPTP) spectroscopies. A brief introduction to these techniques is provided in the second part of this short review.

\section{Ultrafast Light-induced Charge Injection in Solids}

Charge injection from the excited state of a donor molecule into a continuum of electronic acceptor states in a solid has significant importance for the fundamental understanding of the dynamics of electron transfer (ET) processes. Classical theoretical treatments of ET and further quantum mechanical extensions are based on the assumption that the overall ET kinetics are controlled by the nuclear activation barrier to achieve electronic resonance between reactant and product states. The situation can be illustrated by an energy scheme (Fig. 1, left), showing the situation for an ET-reaction between a donor (D) and an acceptor species (A). In a classical view, the system can propagate on the potential energy 
surface (PES) of the electronic configuration, approximated as one-dimensional parabola. Energy conservation allows an electronic transition between the initial encounter complex D-A and the final charge separated state $\mathrm{D}^{+} \mathrm{A}^{-}$only at the intersection of the two PESs, where the electron can be transferred from $\mathrm{D}$ to $\mathrm{A}$.

A fundamentally different situation for electron transfer is found in dye/semiconductor systems. Fig. 1 (right) shows a schematic of such a system. The acceptor level in this case is the energetically broad conduction band of the semiconductor (SC). As a consequence, the final chargeseparated state energy surface splits up into a manifold of acceptor parabolas. In this case, there is no need of an energy matching mechanism via molecular vibrations and the rate constant for interfacial ET is essentially independent of nuclear factors. The only remaining parameter determining the injection rate is the electronic coupling matrix element $|V|^{2}$, representing the electronic overlap integral between donor and acceptor states. ${ }^{[2-4]}$

Recent ultrafast studies have been shown to occur in the femtosecond to picosecond time domain. ${ }^{[3,5-10]}$ Time constants for ET as short as $6 \mathrm{fs}^{[6,7]}$ were found. Charge transfer times $\leq 20$ fs indicate that the reactions occurs on the same time scale or even faster than nuclear motion associated with high-frequency intramolecular vibrations $\left(\tilde{v}>1600 \mathrm{~cm}^{-1}\right)$. The notion that the electron is transferred to the solid well before vibrational relaxation of the photoexcited sensitizer has recently been confirmed in

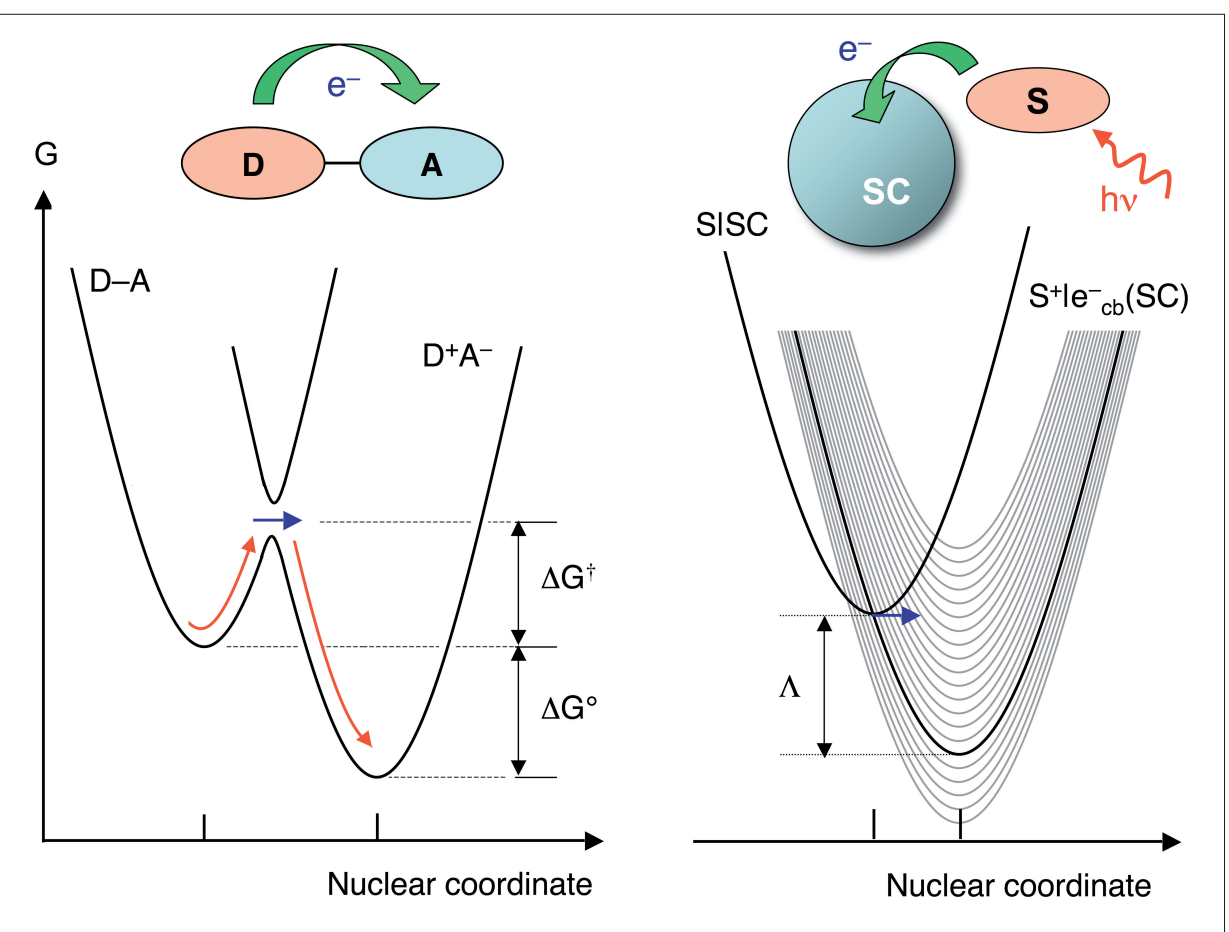

Fig. 1. Energetic situation for a typical molecular electron transfer in the Marcus theory (left) and the situation prevailing in the case of a manifold of acceptor states found in the dye-sensitization of a semiconductor (right) strong coupling cases by the observation of the dependence of ET kinetics upon the excitation photon energy, ${ }^{[4]}$ and that of oscillations in the transient absorption signal due to vibrational wavepacket motion during charge transfer. ${ }^{[7]}$

Because of their successful use in dyesensitized solar cells, Ru(II) polypyridyl complex dyes adsorbed on nanocrystalline $\mathrm{TiO}_{2}$ films are regarded as a model system for the experimental study of the ultrafast dynamics of interfacial light-induced electron transfer. Most studies have reported charge injection kinetics from cis-Ru $\mathrm{RI}^{\mathrm{II}}(\mathrm{dcbpy})_{2}(\mathrm{NCS})_{2}$ (N719) or its protonated form (N3) to take place with a fast (sub-100 fs) phase, followed by a slower (0.7-200 ps) multi-exponential component. ${ }^{[8]}$ Recently, we showed that the observed kinetic heterogeneity can actually result from the aggregation of sensitizer molecules on the surface. A monophasic ET with a rise time shorter than $20 \mathrm{fs}$ is indeed consistently observed when the formation of aggregates is prevented and the sensitizer is adsorbed as a monolayer on the surface of $\mathrm{TiO}_{2}$ nanocrystals. ${ }^{[10]}$

\section{Kinetic Competition between Charge Recombination and Dye Regeneration Processes}

The fate of the charge separated state obtained by injection of an electron from a photosensitizer into the conduction band of the solid, and the prospect to exploit this light-induced interfacial charge separation for practical applications, are essentially limited by the back electron transfer processes that lead to charge recombination. Fig. 2 schematizes the energetics and dynamics of processes that take place after charge injection from a molecular excited state to the acceptor levels of a semiconductor. Thermalization and trapping of hot injected carriers is known to occur typically with a rate constant $k_{t h} \approx 10^{13} \mathrm{~s}^{-1}$. [11] Reverse transfer of a hot electron is therefore generally prevented. The rate of the electron recapture, which takes place between the solid and the oxidized dye species $\mathrm{S}^{+}$ has been observed to be slower by several orders of magnitude compared to charge injection rates of efficient sensitizers. In the $\mathrm{N} 719 / \mathrm{TiO}_{2}$ system, this back electron transfer process typically occurs on a time scale of hundreds of $\mu$ s to ms time scale, $10^{10}$ times slower than the initial photoinduced charge injection. ${ }^{[3,12]}$ At least two main reasons can been invoked to explain such a huge difference:

i) Charge recombination takes place between discrete energy levels and is mediated by vibration energy fluctuations. Its rate is thus scaled down by nuclear factors, which do not intervene in the case of the forward electron transfer process.

ii) While electron injection is kinetically near optimum, the high exoergicity of the back electron transfer can make the system lie deep in the inverted Marcus region, where the rate of the charge transfer process is expected to decrease with increasing driving force. ${ }^{[3,13]}$

The slow charge recombination process can be intercepted by reaction of a reducing mediator D with the oxidized dye (Eqn. (3), Fig. 2). The overall efficiency of the light-induced charge separation then depends upon the kinetic competition between back electron transfer and dye regeneration processes. ${ }^{[14]}$ Photovoltaic cells based on the sensitization of mesoporous titanium dioxide by $\mathrm{Ru}$ (II) complex dyes in conjunction with the $\mathrm{I}_{3}-/ \mathrm{I}^{-}$redox couple as a mediator have proved very efficient at exploiting this principle.[15] Fig. 3 shows, as an example, the temporal evolution of the oxidized state of N719 complex dye sensitizer initially formed during the photoinduced electron injection process and later decaying due to reduction by iodide or charge recombination.

Recently, solid-state devices have been described where the liquid electrolyte present in the pores of the nanocrystalline oxide film is replaced by an organic hole-transporting solid medium containing triarylamine donor functions. Contrary to the case of the oxidation of $\mathrm{I}^{-}$to $\mathrm{I}_{3}^{-}$, which requires the transfer of two electrons, the dye regeneration process in this case is a single electron process and is characterized by a much faster kinetics. ${ }^{[16]}$ 


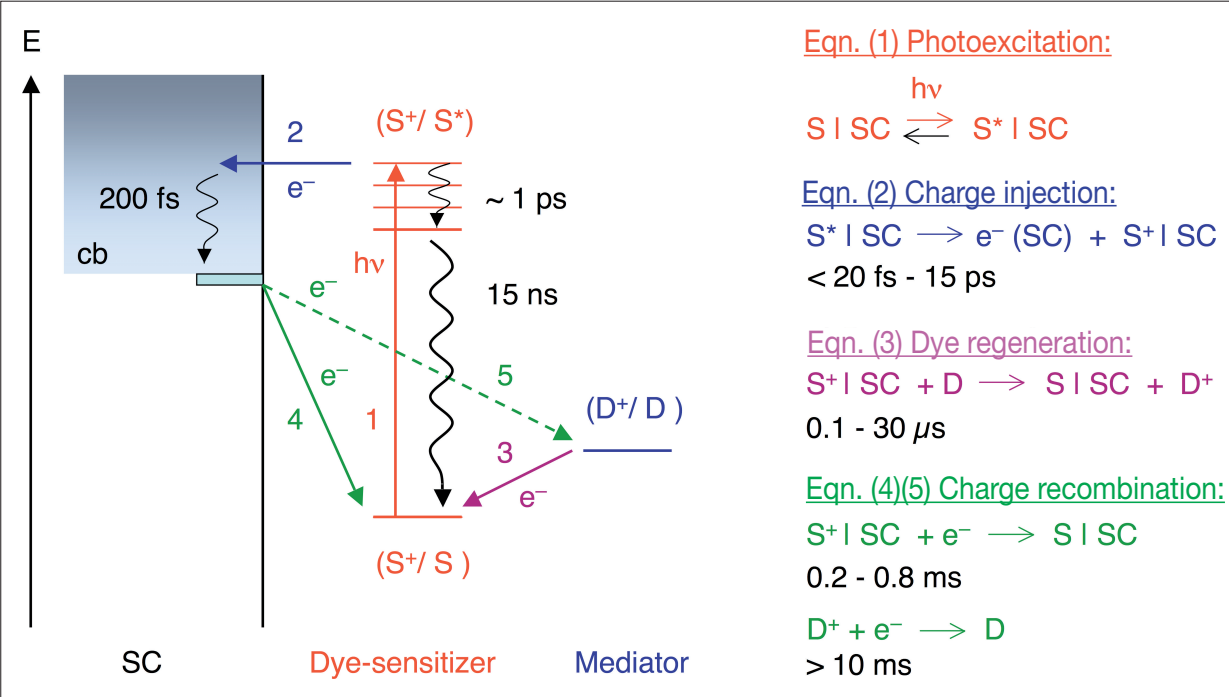

Fig. 2. Energetic scheme of interfacial electron transfer processes following charge injection from the electronic excited state $\mathrm{S}^{*}$ of a dye-sensitizer to the conduction band (cb) of a semiconductor (SC). Typical time frames within which each reaction takes place are indicated for a system constituted of $\mathrm{TiO}_{2}$ nanoparticles, sensitized by N719 dye, in the presence of a concentrated electrolyte containing iodide as a donor (D). Numerical figures show that kinetic competition between ET processes leads to the formation a long-lived charge separated state $e^{-}(c b) . . D^{+}$with almost unit quantum yield.

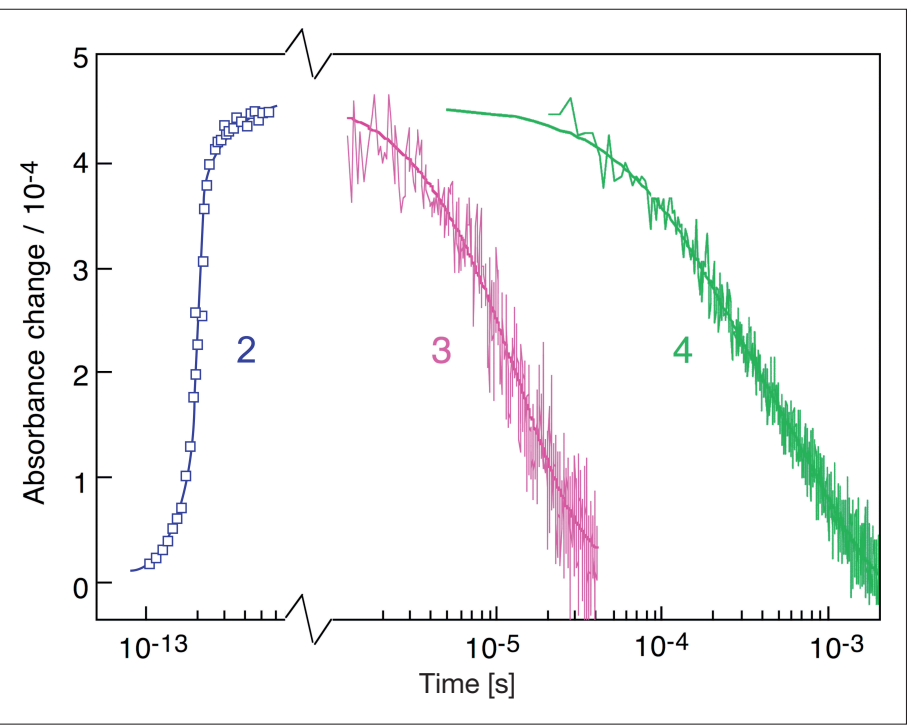

Fig. 3. Transient absorbance signals recorded upon pulsed laser excitation of the N719 dye-sensitizer adsorbed on $\mathrm{TiO}_{2}$ mesoscopic films. Optical signals reflect the appearance and decay of the oxidized state $\mathrm{S}^{+}$of the dye. Data points at the shorter time scale corresponds to the electron injection process and concomittent formation of the $\mathrm{S}^{+}$species (Fig. 2, Eqn. (2)). The decay curve at shorter time scale was obtained in the presence of a liquid electrolyte containing $0.8 \mathrm{M}$ iodide and is indicative of the dye regeneration reaction (Fig. 2, Eqn. (3)). The decay curve at longer time scale is due to the back electron transfer (Fig. 2, Eqn. (4)) and was recorded in pure redoxinactive solvent. Ultrafast transients were measured at a probe wavelength of $860 \mathrm{~nm}$, following pumping at $535 \mathrm{~nm}$. Ns- $\mu$ s data were obtained at $680 \mathrm{~nm}$ upon $600 \mathrm{~nm}$ pulsed laser excitation.

\section{Charge Carrier Dynamics and Transport in Mesoscopic Media}

For $\mathrm{TiO}_{2}$-based nanocrystalline dyesensitized photovoltaic cells, the conversion efficiency to a large extent is limited by electron transport through the oxide structure and conduction of positive charges in the opposite direction via an electrolyte or a hole-transporting material through the pores of the mesoscopic film. ${ }^{[17]}$ Typical charge hopping and scattering times range from femtoseconds to picoseconds, depending on the nature, purity, and temperature of the material. Since these times correspond to the terahertz (THz) frequency range, $\mathrm{THz}$ time-domain spectroscopy (THz-TDS) has emerged as a powerful probe of charge carriers and their transport processes in condensed matter. This method provides direct access to important parameters such as the charge density and scattering times. By combining THz-TDS with synchronous optical excitation, one has optical pump- $\mathrm{THz}$ probe spectroscopy (OPTP) available as a powerful tool for ultrafast time-resolved conductivity studies of materials.[18]

$5 \mathrm{An}$ experimental setup can be built from an existing femtosecond laser system to generate and detect $\mathrm{THz}$ pulses, and then photoexcite a sample and observe the distortion of the transmitted $\mathrm{THz}$ waveform. A schematic view of such a setup is shown in Fig. 4: A chirped pulse amplified laser system provides $\sim 120$ fs pulses at a repetition rate of $1 \mathrm{kHz}$ and at a wavelength of $780 \mathrm{~nm}$. The laser beam illuminates a ZnTe crystal, generating near single-cycle terahertz pulses via optical rectification in this material. The terahertz beam is then focused by gold mirrors onto the sample, and the transmitted beam is refocused onto a second ZnTe crystal constituting the receiver. A portion of the laser red light is split off, passed through the receiver crystal and converted to circularly polarized by a $\lambda / 4$-plate. Using a polarizing beamsplitter, the two orthogonal components of the circularly polarized light are then separated and subtracted using a pair of balanced diode photodetectors (the net current for circularly polarized light is zero). The terahertz beam transmitted by the sample induces a birefringence in the ZnTe crystal of the receiver (Pockels effect) which rotates the polarization of the $780 \mathrm{~nm}$ beam such as, after the $\lambda / 4$-plate, it is elliptically polarized. The resulting signal provided by the balanced photodetectors is then measured by a lock-in amplifier referenced to the chopper wheel. The receiver is gated synchronously with the transmitted $\mathrm{THz}$ beam, and by varying the delay line one can map out the complete time dependent electric field.

In addition to electronic conduction in the condensed phase, THz-TDS can probe long-range crystalline lattice vibrations, low energy torsion and hydrogen bonding vibrations. Phonons associated with the self trapping of photo-generated or injected carriers (small polarons) are detected and analyzed in real time, allowing in particular a detailed study of vibrational coherence associated with electron transfer processes at dye-sensitized interfaces. ${ }^{[7]}$

\section{Acknowledgement}

Many molecular systems and materials used in our studies are kindly provided by the Laboratory for Photonics and Interfaces (LPI) of EPFL. Fruitful collaboration in particular with P. Comte, S. M. Zakeeruddin, M. K. Nazeeruddin, and M. Grätzel of LPI is gratefully acknowledged. 


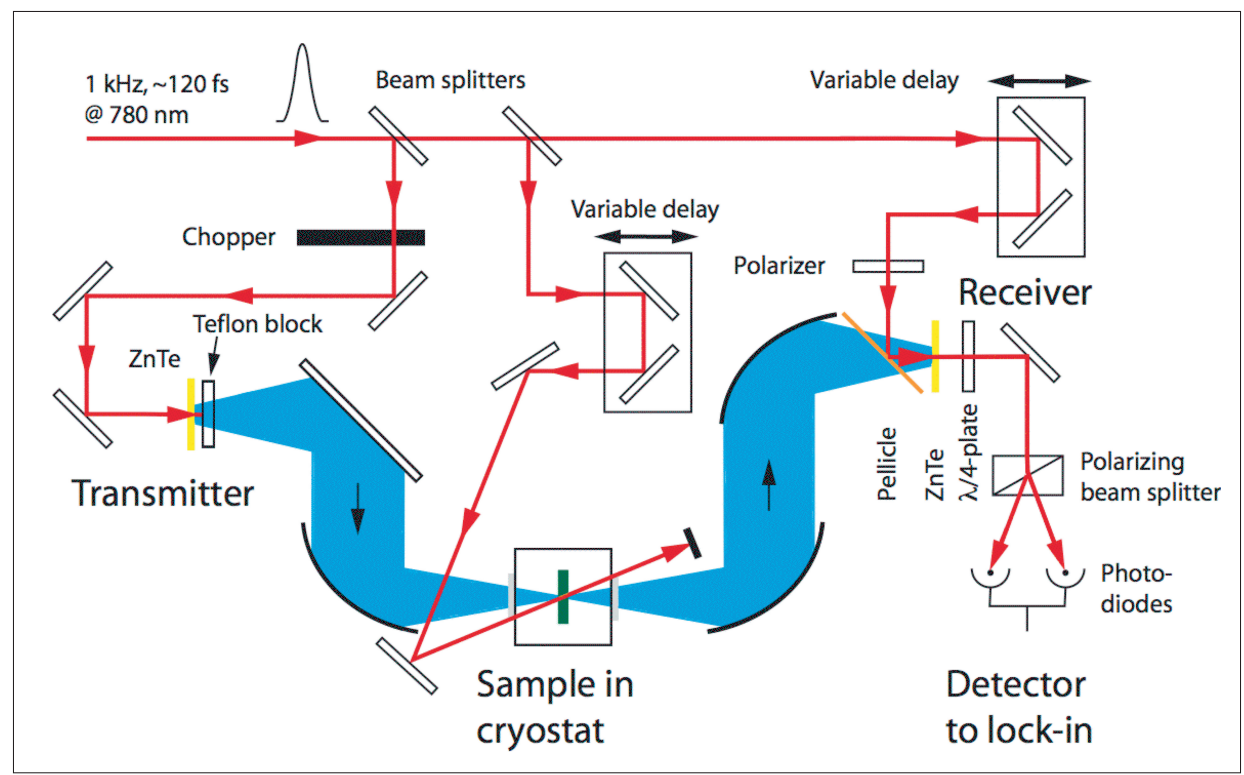

Fig. 4. Schematic view of the terahertz time-domain spectroscopy (THz-TDS) experimental setup. An additional pump beam, either provided by splitting off part of the fundamental red femtosecond laser ray or by tuning the laser wavelength in an optical parametric amplifier (OPA), allows experiments to be carried out in an optical pump-terahertz probe (OPTP) configuration.

This research is made possible thanks to the financial support of the Swiss National Science Foundation, an EC Marie Curie International Reintegration Grant and scientific equipment funding by EPFL.

Received: July 27, 2007

[1] M. Grätzel, in 'Handbook of nanostructured materials and Nanotechnology', Ed. H. S. Nalwa, Academic Press, New York, 2000, Vol. 3.

[2] a) J. M. Lanzafame, S. Palese, D. Wang, R. J. D. Miller, A. A. Muenter, J. Phys. Chem. 1994, 98, 11020; b) R. J. D. Miller, G. L. McLendon, A. Nozik, W. Schmickler, F. Willig, 'Surface Electron-Transfer Processes', VCH, New York, 1995.

[3] M. Grätzel, J. E. Moser, 'Solar Energy Conversion', in 'Electron Transfer in Chemistry', Ed. V. Balzani, Wiley-VCH, Weinheim, 2001, Vol. 5, pp 589.

[4] a) J. E. Moser, M. Grätzel, Chimia 1998, 52, 160; b) J. E. Moser, M. Wolf, F. Lenzmann, M. Grätzel, Z. Phys. Chem. 1999, 212, 85.

[5] J. E. Moser, P. Bonhôte, M. Grätzel, Coord. Chem. Rev. 1998, 171, 245.

[6] R. Huber, J. E. Moser, M. Grätzel, J. Wachtveitl, J. Phys. Chem. B 2002, 106, 6494.

[7] R. Huber, J. E. Moser, M. Grätzel, J. Wachtveitl, Proc. SPIE 2003, 5223, 121.

[8] Y. Tachibana, J. E. Moser, M. Grätzel, D. R. Klug, J. R. Durrant, J. Phys. Chem. 1996, 100, 20056.

[9] S. Pelet, M. Grätzel, J. E. Moser, J. Phys. Chem. B 2003, 107, 3215.

[10] a) B. Wenger, M. Grätzel, J. E. Moser, J. Am. Chem. Soc. 2005, 127, 12150; b) D. Kuang, S. Ito, B. Wenger, C. Klein, J.-E. Moser, R. Humphry-Baker, S. M. Zakee- ruddin, M. Grätzel, J. Am. Chem. Soc. 2006, 128, 4146.

[11] D. P. Colombo, R. M. Bowman, J. Phys. Chem. 1996, 100, 18445.

[12] S. Haque, Y. Tachibana, R. L. Willis, J. E. Moser, M. Grätzel, D. R. Klug, J. R. Durrant, J. Phys. Chem. B 2000, 104, 538.

[13] J. E. Moser, M. Grätzel, Chem. Phys. 1993, 176, 493.

[14] a) S. Pelet, J. E. Moser, M. Grätzel, J. Phys. Chem. B 2000, 104, 1791; b) H. Nusbaumer, J.-E. Moser, S. M. Zakeeruddin, M. K. Nazeeruddin, M. Grätzel, $J$. Phys. Chem. B 2001, 105, 10461.

[15] a) M. Grätzel, Nature 2001, 414, 338; b) P. Wang, S. M. Zakeeruddin, J. E. Moser, M. K. Nazeeruddin, T. Sekiguchi, M. Grätzel, Nature Mater. 2003, 2, 402.

[16] U. Bach, D. Lupo, P. Comte, J. E. Moser, F. Weissörtel, J. Salbeck, H. Spreitzert, M. Grätzel, Nature 1998, 395, 583; b) J. E. Moser, Nature Mater. 2005, 4, 723.

[17] N. Kopidakis, E. A. Schiff, N. G. Park, J. van de Lagemaat, A. J. Frank, J. Phys. Chem. B 2000, 104, 3930.

[18] a) R. D. Averitt, A. I. Lobard, C. Kwon, S. A. Trugman, V. K. Thorsmølle, A. J. Taylor, Phys. Rev. Lett. 2001, 87, 017401; b) V. K. Thorsmølle, R. D. Averitt, X. Chi, D. J. Hilton, D. L. Smith, A. P. Ramirez, A. J. Taylor, Appl. Phys. Lett. 2004, 84, 891. 